\title{
Implementing a Flexible Testbed Using Dynamic FFR Scheme in OFDMA Based Cognitive Radio Networks
}

\author{
Joydev Ghosh ${ }^{1}$, Sanjay Dhar Roy ${ }^{2}$ \\ ${ }^{l}$ ETCE Dept., The New Horizons Institute of Technology, Durgapur-8, W.B, INDIA \\ E-mail: joydev.ghosh.ece@gmail.com \\ ${ }^{2}$ ECE Dept., National Institute of Technology, Durgapur-9 (W.B), INDIA \\ E-mail : s_dharroy@yahoo.com
}

\begin{abstract}
In this paper, the femtocell resource allocation in the Orthogonal Frequency Division Multiple Access (OFDMA) integrated cognitive radio (CR) networks has been demonstrated to optimize the network performance, while reducing the outage users at cell edges. The sub-band sharing flexibility not only improves the spectral efficiency of the dynamic FFR scheme against conventional approach but also it alleviates outage probability and further noticeable amount of improvement can be found in superior performance parameters such as capacity, CDF of SINR and overall cell throughput. We yield an analytical framework to ascertain the orthogonality of the subcarriers by compensating carrier frequency offset (CFO) to ensure better OFDMA performance in the proposed network model and illustrates the influence of frequency reuse factor (FRF) at cell edges via simulation results.
\end{abstract}

Keywords-CR-femtocell Networks; OFDMA scheme; Fractional Frequency Reuse (FFR) Scheme; Frequency Reuse Factor (FRF)

\section{INTRODUCTION}

The loads on cellular networks are enhancing as both the number of users and the communication demands per user increased. In the latest cell design for communication systems, the growing body of users and hence the enhancing levels of interference-will eventually deluge base stations' abilities to communicate with everybody simultaneously and reliably. Nowadays, quite a few solutions are existing to tackle with this growth and one quite obvious approach towards solution can be by simply adding more spectrums. It is not feasible in practice due to commercially available spectrum is already used up, so other way out is to utilize smaller cells. It aids the matter by allocating minimum number of users to individual base station, so that, decreases the load and intra-cell interference (ICI) in each channel. Research reveals that up to $70 \%$ of entire mobile data need is at present generated from indoor [1]. The high indoor data rate is hard to achieve in $4 \mathrm{G}$ because of large wall penetration losses result in week received signal strengths (RSS). The complication is especially acute in cell-edge areas furthest from the serving macrocell base stations (MBS). A bigger part of the allotted band in distinct networks is yet not efficiently utilized and it results in under-utilization of a noticeable amount of frequency band [2]. The solution of this problem has introduced the idea of CR technology [3]. The CR technology has capability to improve the quality of spectrum usage by detecting the spectrum holes that are underused by licensed primary users (PUs). The allocation of unused spectrums is employed on unlicensed secondary users (SUs) who need more spectrum resources. The SUs are getting the provision to communicate concurrently with the PUs in the same spectrum by the existing spectrum sharing technique which is called spectrum underlay [5]. Hence, a CR network could be drawn in a way that the quality of service (QoS) demands of the SUs is fulfilled, while make sure that the limitation of interference power of primary radio network (PRN) is fulfilled.

Multicarrier networks can furthermore assist in optimizing the use of spectrum as they permit to put the carriers in a noncontiguous manner. An abstract of several multicarrier modulation techniques with regard to the optimum utilization in CR networks is described in [4]. The salient properties and the potential of the OFDMA technology makes it perfectly suitable for the CR based communication network. The prime concept of OFDMA scheme is that the accessible spectrum splits into many orthogonal sub-channels and allows more than one users to transmit data concurrently on the distinct sub-channels. But the Doppler frequency offset in high mobility scenarios, where the pilot channel fluctuates at the time of communication, destroys the orthogonality of subcarriers [8]. And this is the reason for which OFDMA is so sensitive to the inter carrier interference (ICI). The incorporation of CR technology and OFDMA is one of the best properties in next generation networks because of its adaptability in assigning resource among CR users [5]. The slow fading environment has been studied in [6] to analyze the SUs interference for cognitive radio (CR) technology.

The network model in this article also presumes that FBSs are furnished with CR potentiality. The distribution of smallpower base station within the coverage of a macrocell network makes the entire network heterogenous in nature. The FFR scheme has been introduced as a productive inter cell interference coordination (ICIC) method in an OFDMA dependent wireless network. The use of FFR scheme in 
heterogenous networks (HetNets) reduces compromise among data rate, network coverage for the outage users, macrocell throughput and spectral efficiency (SE). If the formation of FBSs grows, the probability of assigning the same spectrum to two neighbour femtocells grows, and the inter small cell interference even more important in this regards [9]. Carrier frequency offset (CFO) is one of the main reasons of spectrum mispositioning between transmitter and receiver filter. This mispositioning creates energy loss and distortion in the received signal, resulting in performance deterioration of the entire network [10]. It also causes ICI between sub-carriers, which dismantle the orthogonality of sub-carriers that cause deterioration in bit error rate (BER).

In this paper, we initially compensated carrier frequency offset (CFO) to maintain orthogonality of the subcarriers to ensure good performance of OFDMA scheme in the proposed network model. Thereafter, we have deployed more number of FBS at the cell edge area through dynamic FFR algorithm [7]. The users located far from macro base station (MBS) positioned at $(0,0)$ co-ordinates, more precisely, the users close to the macrocell edge are aided by cognitive-femto base station (FBS) result in reliable quality of service (QoS). Frequency reuse scheme is one of the solutions to help the network optimization, it split whole frequency spectrum into quite a few sub-bands and every sub-band is separately allotted to middle area and edge area of the macrocell. Frequency Reuse Factor (FRF) of middle area is 1, whereas, edge area associates higher frequency reuse factor, 3 . We sort out the optimization difficulty by assigning frequency subbands to FBS in each area and compute a productive interference co-ordination scheme utilizing sectored-FFR and frequency spectrum allocation. FFR applies to the macrocell for the purpose of frequency spectrum allocation and simultaneously, femtocells will get the opportunity to select sub-bands which are underutilized in the sub-zone of macrocell to circumvent interference. Here, objective is to maximize SINR of user with the consideration of fairness. Our main focus is on the downlink data service issue. Our main contributions can be summarized as follows-

- The accurate channel state information (CSI) with minimum ICI is estimated by taking the analytical measure of channel interference ratio (CIR).

- Improvements in network coverage and outage performance of proposed networks are being achieved through the deployment of large number of femtocells at cell edge area. The effect of SINR and center radius ratio on channel capacity for $\mathrm{FRF}=1$ and 3 are computed by dynamic FFR algorithm at cell edges [7]. The centre radius ratio is defined as the ratio of the distance from the centre of a user to the radius of the macrocell .

- The effect of large number of FBS implantation and higher FRF effect on the CDF of SINR by modulation and coding scheme (MCS) selection for a number of users in OFDMA based cognitive femtocell networks have been formulated.

- We present comprehensive numerical outcome to validate the developed simplified network model and to exhibit the effectiveness of our proposed scheme for different parameter settings.

The remainder of this paper is organized as follows. Section II depicts system model and OFDMA analysis of downlink (DL) transmission. Section III presents simulation model. Then, Section IV provides simulation results. Finally, Section V concludes this paper.

\section{SYSTEM MODEL}

In this paper, DL-OFDMA transmission with one hexagonal grid macrocell and $\mathrm{N}_{\mathrm{F}}$ femtocells deployed in macrocell coverage area has been demonstrated. As depicted in Fig.1, a MBS is located at middle of the network and a number of FBSs, $N_{F}=12$ is implanted at the macrocell edge area. In the network coverage of MBS, a total number of mobile users denoted by $N_{M U}$ are randomly distributed. Similarly, entire $N_{F U}$ number of users is randomly deployed in $j$-th femtocell, $j \in \mathrm{N}_{\mathrm{F}}=\left\{1,2, \ldots, N_{F}\right\}$. Let us assume that $F A P_{j}$ and $F U_{j}^{(i)}$ be the $j$-th FAP and the $i$-th user in the $j$-th femtocell, respectively. The resource blocks (RBs) are issued uniformly among the MUs in a macrocell and the same RBs are again

\footnotetext{
FBS Coverage $=$ Green Circle Inner Region =Red Circle; User=Gray bubble
}

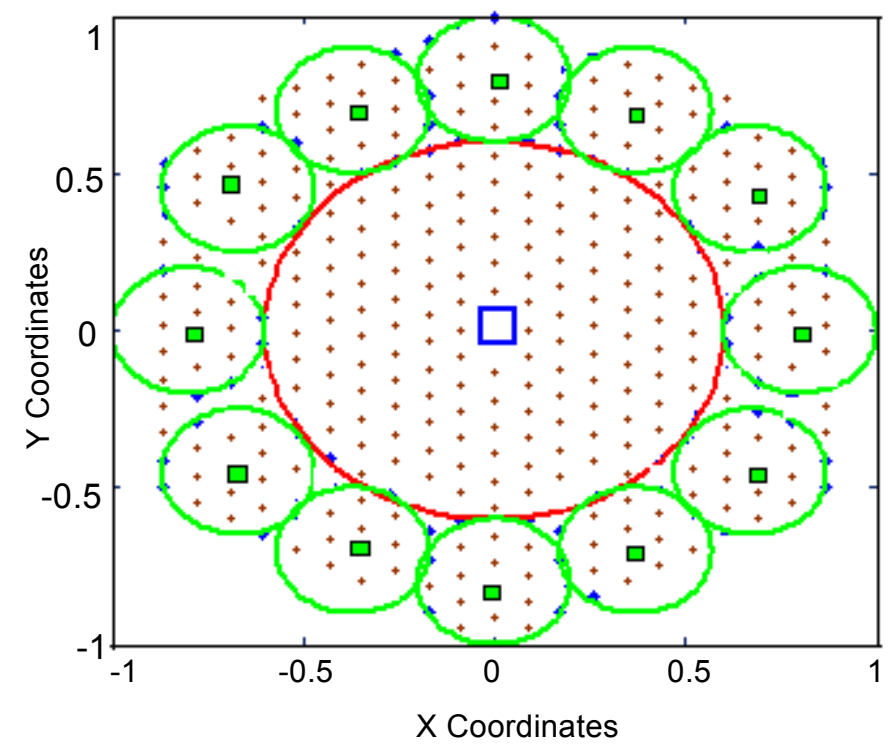

Fig.1 A densely deployed femtocell scenario in the coverage of the macrocell network.

reused by each femtocell. In order to do fair contrast, the assessment of transmit power for each RB is made a priori and it is to be kept unchanged for entire network [8]. We consider that there is a total $N_{R B}$ number of RBs in the entire network. In this place, the macrocell takes priority over the femtocell to determine the number of RBs to serve its own users, i.e., $N_{M R B}$. The rest $\left(N_{R B}-N_{M R B}\right)$ RBs are orthogonally allocated to femtocells. Therefore, $\left[\left(N_{R B}-N_{M R B}\right) / N_{F}\right] \mathrm{RBs}$ are allocated to individual femtocell. FFR scheme is applied in the macrocell, where the total spectrum correlated to the macrocell edge area is split up into 3 sub-bands. A sector is allocated to single sub-band consisting $N_{s c}$ number of subchannels that are accessible for both the user associated with the cell-centre and the user associated with the edge area of macrocell to prop up. Every sub-channel (SC) indicates a fundamental time frequency unit. The coverage of femtocell is 
$R_{f}$ and the distance between adjacent FBSs is $d_{f b s}^{f b s}$. We consider that each FBS is positioned at the centre of rooms and assigns $\left[\rho_{f} N_{s c}\right]$ sub-channels to its serving FUs. Here, the sub-channel utilization ratio $\rho_{f}$ is considered as the ratio of the utilized sub-channels to the entire sub-channels allocated to each SB. The typical value of $\rho_{f}$ lies in between 0 and 1 . Every sub-channel is assigned to a single user utmost within the same cell and could be shared in separate cell. So that, cotier interference is eliminated and only cross-tier interference is assumed. We consider that MBSs transmit equal power over all sub-channels, where FBSs can manage their transmit powers to adapt to the practical scenario.

\section{A) Weighting Co-efficient Effect and CFO on ICI in standard OFDMA system-}

The OFDMA modulator normally composed of an N-point Inverse Discrete Fourier Transform (IDFT) and a unit to include the guard interval (cyclic prefix). The IDFT processing is expressed by the IDFT matrix:

$$
I D F T=\left\{b^{l k}\right\} \in C^{N x N} \quad b=\exp (j 2 \pi / N)
$$

The inclusion of the cyclic prefix (CP), which is composed of $\mathrm{G}$ samples, is achieved by means of the guard interval matrix:

$$
G I=\left[\begin{array}{cc}
0 & I_{G x G} \\
I_{N \times N} & 0
\end{array}\right] \in N^{(N+G) x N}
$$

where I is the identity matrix. We construct the signal vector $\mathbf{s}$ :

$$
\boldsymbol{s}=\text { GI.IDFT.A. } \boldsymbol{x}
$$

where the element $\boldsymbol{x}$ is fetching the pilots together with the essential data. The symbol $x_{i}$ represents the transmitted signal vector $\boldsymbol{x} \in C^{N^{\prime}}$. The length $N^{\prime}$ of the transmitted signal vector is adapted before the IDFT signal processing. The system of ascertaining the right placement of $\boldsymbol{x}$ at the IDFT input is done by means of the matrix $A \in N^{N x N^{\prime}}$. The symbol $S_{i}$ is conveyed in a series over the mobile channel.

In general, the received signal in OFDMA receiver is crossbreeding with a local oscillator signal where carrier frequency is lesser than the received signal by the amount of $\Delta f$. After considering $\mathrm{N}$ point DFT of the signal at demodulator, the demodulated element at $m$-th sub-carrier of $i$ -th OFDMA symbol can be expressed by [11]:

$$
z_{m, i}=\exp \left(j\left(\theta_{o}+(i-1) \emptyset\right)\right) \sum_{l=0}^{N-1} c_{l-m} a_{l, i}
$$

where the weighting co-efficient, $c_{l-m}$, can be expressed by [12]:

$$
\begin{aligned}
& c_{l-m}=\frac{1}{N} \sum_{k=0}^{N-1} \exp \left(\frac{j 2 \pi k(l-m+\varepsilon)}{N}\right) \\
& =\frac{1}{N} \frac{\sin (\pi(l-m+\varepsilon))}{\sin \left(\frac{\pi(l-m+\varepsilon)}{N}\right)} \\
& \times \exp \left(j \pi\left(\frac{N-1}{N}\right)(l-m+\varepsilon)\right)
\end{aligned}
$$

and $a_{l, i}$ represents the QPSK symbol conveyed from the $l$-th subcarrier of the i-th OFDMA symbol. The symbol $\theta_{o}$ indicates phase component between the local oscillator signal and the carrier signal. $\varnothing=2 \pi \varepsilon(1+G / N)$ is the phase rotation produced by the $\mathrm{CFO}$ in which $\varepsilon=f_{d(\max )} T_{s y s}=$ $f_{d(\max )} / f_{\text {sub }}$ is the normalized CFO which describes the timevarying characteristics of the channel and $f_{\text {sub }}$ represents the subcarrier spacing. The typical value of $|\varepsilon|<0.5$.

The Doppler frequency component has to be lesser than:

$$
f_{d(\max )}=\frac{1}{\left(2 \cdot N_{t} \cdot T_{s y s} \cdot(1+G)\right)}
$$

where $T_{s y s}$ is the duration of one OFDMA symbol and it can further be defined as $T_{\text {sys }}=N . T_{s}$ in which $T_{s}$ denotes sampling interval, $\mathrm{G}$ guard interval factor and $N_{t}$ is the pilot spacing in time-direction.

It can be observed that demodulated complexed value $Z_{m, i}$ composed of a required element due to $a_{m, i}$ as well as other components due to the interference. Hence,

$$
z_{m, i}=\exp \left(j\left(\theta_{0}+(i-1) \emptyset\right)\right)\left(c_{0} a_{m, i}+u_{m, i}\right)
$$

where $u_{m, i}=\sum_{l=0, l \neq m}^{N-1} c_{l-m} a_{l, i}$

is the ICI for the m-th sub-channel and normalized weighting co-efficient,

$$
c_{0}=\frac{\sin (\pi \varepsilon)}{(\pi \varepsilon)}=\frac{1}{\Gamma(1+\varepsilon) \Gamma(1-\varepsilon)}
$$

Quite a few of isolated pilot tones is incorporated in the received stream to estimate the $\mathrm{CFO}$ to ignore the effect of ICI. Equation no. (7) signifies that each and individual demodulated element is corrupted by ICI from all other subchannels. Once the offset value is estimated, it will be returned to the input end of the receiver to rectify the received signal.

Considering that one of the pilot tones is the m-th subcarrier which scatters over the entire spectrum, the decoded element of this tone in the $(i+1)$ th OFDMA symbol is expressed by [13]:

$$
z_{m, i+1}=\exp \left(j\left(\theta_{0}-i \emptyset\right)\right)\left(c_{0} a_{m, i+1}+u_{m, i+1}\right)
$$

An estimate of CFO is calculated using the m-th pilot tone from a block of L symbols. Based on [14], [15], phase can be calculated using $\mathrm{m}$-th pilot tone from a block of $\mathrm{L}$ symbols as

$$
\tan ^{-1} \frac{\operatorname{Im} \sum_{i=1}^{L-1} z_{m, i}^{*} z_{m, i+1}}{\operatorname{Re} \sum_{i=1}^{L-1} z_{m, i}^{*} z_{m, i+1}}
$$

Therefore, normalized CFO can be estimated by [16]

$$
\begin{aligned}
& \widehat{\varepsilon_{m}}=\frac{1}{2 \pi(N+G) / N} \tan ^{-1} \frac{\operatorname{Im} \sum_{i=1}^{L-1} z_{m, i}^{*} z_{m, i+1}}{\operatorname{Re} \sum_{i=1}^{L-1} z_{m, i}^{*} z_{m, i+1}} \\
& =\frac{1}{2 \pi(1+G / N)} \tan ^{-1} \frac{\operatorname{Im} \sum_{i=1}^{L-1} z_{m, i}^{*} z_{m, i+1}}{\operatorname{Re} \sum_{i=1}^{L-1} z_{m, i}^{*} z_{m, i+1}}
\end{aligned}
$$

Including all the pilot tones in account, $\bar{\varepsilon}=\frac{1}{P_{t}} \sum_{m=1}^{P_{t}} \hat{\varepsilon}_{m}$, where $P_{t}$ represents the total number of pilot tones. 
Equation no. (11) signifies that the accomplishment of the CFO is dependent on the two consecutive OFDMA symbols such as $Z_{m, i}^{*} z_{m, i+1}$. The variance of the phase rotation estimate from the two consecutive OFDMA symbols including slow fading environment is expressed by [12]

$$
\operatorname{var}(\varnothing)=\frac{1}{(L-1)^{2}}\left(\frac{1}{C I R}+\frac{L-1}{2} \frac{1}{C I R^{2}}\right)
$$

where channel interference ratio (CIR) [28] is

$$
C I R=\frac{\left|c_{m}\right|^{2}}{\sum_{l=1}^{N-1}\left|c_{m, l}\right|^{2}} \quad \forall m \in 0,1,2,3 \ldots . .
$$

From (13), it is seen that the lower CIR consequently results in a higher variance.

\section{B) Downlink (DL) SINR Estimation-}

The link gain in double-tier networks can be expressed by [17]-

$$
G=d^{-\alpha_{p}} 10^{\xi_{s}} / 10|h|^{2}
$$

where $j$-th FBS and $i$-th FU are separated by the location gap, $d$, a Gaussian random variable with 0 mean, $\xi_{\mathrm{s}}$ (in $\mathrm{dB}$ ) and $6^{2}$, variance, because of shadowing in the channel. Here $|h|^{2}$ indicates the gain of the channel created between $k$-th MBS and respective $i$-th FU. Besides, Rayleigh fading provides tractable outcomes that aids to understand the network response to certain conditions. We put to use the symbol $x$ which indicates the network entity which provides service for a user. This means, $x=f$ if the user associated with FBS and $x=m$ if the user associated with MBS. Hence SINR, $\gamma_{n, i, k}^{x}$ of a typical user, located at the centre (that also holds true for a generic user), be in the service of the MBS or FBS can be expressed by [18]-

$$
\gamma_{n, i, k}^{x}=\frac{P_{n, i, k}^{x} G_{n, i, k}^{x}}{I_{n, i, k}^{x *}+I_{n, i, k}^{\prime x *}+\sigma_{n, i, k}^{2}}
$$

where $G_{n, i, k}^{x}$ is the channel gain between the user to the network entity which provides service (i.e. either a MBS or a FBS) on top of the $n$-th sub-channel. Here $P_{n, i, k}^{x}$ is indicated as a part of total transmit power by the corresponding network entity which provides service on top of the certain subchannel. Similarly, gain of the channel from a centre position $x \in \mathbb{D}^{2}$ to $\mathrm{MBS}, m_{i}$ and $\mathrm{FBS}, f_{i}$ are symbolized by $h_{b_{i}} \sim \sqrt{X_{m_{i}}^{2}+Y_{m_{i}}^{2}}$ and $h_{a_{i}} \sim \sqrt{X_{f_{i}}^{2}+Y_{f_{i}}^{2}}$, respectively, where $X_{x}, Y_{x}$ are designated as independent gaussian random variables with zero mean and required variance, additive white gaussian noise (AWGN) can be symbolized for noise power by $\sigma_{n, i, k}{ }^{2}$.

C) Channel Capacity-Now we estimate the channel capacity as per Shannon's theorem. The channel capacity (after considering FRF) [19] may be expressed as:

$$
C=\left(\frac{B W}{F R F}\right) \cdot \log _{2}(1+S I N R) b p s / H z
$$

D) Cell Throughput-Strong received signal power is feasible to achieve for shortest distance between the transmitter $\left(T_{X}\right)$ and the receiver $\left(R_{X}\right)$ as well as longest distance between the interference source and receiver $\left(R_{X}\right)$, this results in high SINR performance and less number of outage users. So that, it's quite natural to have low throughput at macrocell border area.

$$
\text { Overall Cell Throughput }=\text { Cell Throughput } \text { center } \text { area }^{+}
$$$$
\text { Cell Throughput } \text { edge area }
$$

The corresponding spectral efficiency of user $i$ served by the service network entity, FBS $j$, referred to as user $j(i)$ through the $n$-th sub-channel can be expressed as

$$
C_{j(i)}^{n}=f_{M C S}\left(\operatorname{SINR} R_{j(i)}^{n}\right)
$$

where $f_{M C S}($.$) signifies a function that maps the SINR to the$ largest achievable rate for given modulation coding scheme (MCS) set.

The outage probability of user $j(i)$ through the $n$-th subchannel can be expressed as

$$
\breve{P}_{n, j(i)}=P_{r}\left\{\operatorname{SINR} R_{n, j(i)} \leq S I N R_{T h}\right\}
$$

where $S I N R_{T h}$ is the minimum SINR to achieve the target data rate for a given MCS table.

The research on this issue is attempted to improve the overall cell throughput by improving cell throughput to the edge area through densely deployed femtocell. Cell Throughput is one of the prime parameters to take the measure of performance of femtocells embedded network.

\section{TABLE I: MODULATION CODING SCHEME (MCS) TABLE [20]}

\begin{tabular}{|c|c|c|c|c|}
\hline Index & SINR range dB & Modulation & Code rate & Bit rate \\
\hline 1 & $-3.14 \leq$ SINR $<-0.73$ & QPSK & $1 / 12$ & 1.6 \\
\hline 2 & $-0.73 \leq$ SINR $<2.09$ & QPSK & $1 / 6$ & 3.2 \\
\hline 3 & $2.09 \leq$ SINR $<4.75$ & QPSK & $1 / 3$ & 6.4 \\
\hline 4 & $4.75 \leq$ SINR $<7.86$ & QPSK & $1 / 2$ & 9.6 \\
\hline 5 & $7.86 \leq$ SINR $<9.94$ & QPSK & $2 / 3$ & 12.8 \\
\hline 6 & $9.94 \leq$ SINR $<13.45$ & $16 \mathrm{QAM}$ & $1 / 2$ & 19.2 \\
\hline 7 & $13.45 \leq$ SINR $<18.6$ & 16QAM & $2 / 3$ & 25.6 \\
\hline 8 & $18.6 \leq$ SINR $<24.58$ & 64QAM & $2 / 3$ & 38.4 \\
\hline 9 & $24.58 \leq$ SINR & 64QAM & $5 / 6$ & 48 \\
\hline
\end{tabular}

\section{Simulation Model}

The simulation is developed in MATLAB. In our simulation, parameters mentioned in Table II is used. In particular, each simulation run is carried out according to the following steps.

1. A fixed number of users, $N_{U E}=N_{M U, u}+N_{F U, j i}$ is produced and they are randomly deployed within the coverage area of macrocell. This contains entire MUs /PUs $\left(N_{M U, u}\right) \quad$ and FUs/SUs $\left(N_{F U, j i}\right)$. Here, $j \in N_{F}=$ $\left\{1,2, \ldots, N_{F}\right\} . m=100, u \in\left\{1,2,3 \ldots \ldots \mathrm{mR}_{\mathrm{b}}\right\}, R_{b} \in$ No. of resource block (RB); $i \in\{1,2,3, \ldots . L\}, \forall L$.

2. The positions (in the $(r, \theta)$ co-ordinate system) of entire MUs and FUs $\left(N_{U E}\right)$ are produced within the network coverage area of macrocell. $\mathrm{N}_{\mathrm{FUE}, \mathrm{ji}}$ number of FU receivers are produced around $\left(N_{U E}\right)$ number of corresponding $\mathrm{FU}$ transmitters (FBS/FAP). The FU receivers are present around a FU transmitter, within a circle of radius, $r_{\text {femto }}$. 
3. The distance of each FBS is computed in reference to MBS, which is located at $(0,0)$ co-ordinate of proposed network model. How far MBSUs and FBSUs are located form MBS and FBS, respectively, also computed concurrently.

4. The quantity of useful data bits is liable to vary, which is determined by the modulation coding scheme (MCS). The utilization of MCS is explicated by the link adaptation technique. The choice of suitable MCS is dependent on the value of the receiver SINR. Beside this, MCS is adjusted at the BS emulator. The data rate is a function of SINR for different MCS assuming an ITU pedestrian B radio channel [21]. For very low SINR of up to $9.94 \mathrm{~dB}$ QPSK $2 / 3$ is the best choice and provides a data rate of about 12.8 Mbps. From $9.94 \mathrm{~dB}$ to $13.45 \mathrm{~dB}$ SINR the highest data rate up to $19.2 \mathrm{Mbps}$ is achievable by means of 16-QAM $1 / 2$. The 64-QAM $2 / 3$ offers the best result for SINR from $13.45 \mathrm{~dB}$ to $18.6 \mathrm{~dB}$, while for very good interference conditions with more than $18.6 \mathrm{~dB}$ to $24.58 \mathrm{~dB}$ SINR, 64QPSK 5/6 provides maximum data rate of about 38.4 Mbps. Normally, subscriber switches to a large energy efficient MCS if the SINR is high. The subscriber can also switch to even more robust MCS if the SINR is small. Once the MCS is determined, the amount of bits per symbol can be computed.

5. Normally, subscriber switches to a large energy efficient MCS if the SINR is high. The subscriber can also switch to even more robust MCS if the SINR is small. Once the MCS is determined, the amount of bits per symbol can be computed.

\section{RESULTS AND DISCUSSION}

The accomplishment of the proposed network model is verified by the simulation framework with the use of MCS table in Table I. The common simulation parameters are summarized in Table II.

Fig.2a illustrates that the magnitude of the second order difference of the weighting coefficient can greatly increased for higher CFO $(\varepsilon)$. The property of antipodal data symbols at each pair of subcarriers has used in [24] to mitigate the impact of ICI by means of sacrificing one half of the bandwidth.

Fig.2b shows the channel interference ratio (CIR in $\mathrm{dB}$ ) as a function of normalized CFO $(\varepsilon)$, where $N=32$. The comparison affirms the greater CIR of the weighting coefficient because of self-ICI cancellation.

In Fig.3a, the downlink channel capacity is shown as a function of SINR for different FRF of cognitive radio dependent macrocell network where femto base station (MBS) users are referred as secondary users (SUs). In Fig.3b, the downlink channel capacity is shown as a function of center radius ratio for different $\mathrm{FRF}$ of cognitive radio based macrocell network where femto base station (MBS) users are referred as secondary users (SUs). The result in Fig.3b shows that the downlink (DL) channel capacity decreases with increase in center radius ratio for different FRF, whereas the downlink (DL) channel capacity increases with increase in SINR illustrated in Fig.3a.

In Fig.4, the overall cell throughput is shown as a function of center radius ratio, where the overall cell throughput significantly improved $\mathrm{FRF}=3$ in contrast to $\mathrm{FRF}=1$ at the macrocell cell edge area due to the application of dynamic FFR algorithm.
Fig.5a shows the cummulative distribution function (CDF) of the SINR of the proposed networks when the values of FRF are 1 and 2, respectively, the number of femtocells is 20 and the sub-channel usage ratio $\boldsymbol{\rho}_{f}=\mathbf{1}$ (i.e., the FBS utilizes entire sub-channels in the operating sub-band (SB)). The proposed network model achieves higher average user SINRs for $F R F=3$ in contrast to the conventional $F R F=1$, where each base station (BS) uses the entire network bandwidth for transmission and there is no such frequency drafting among the cells to cope up with the interference from adjacent cells. Fig.5b shows the cummulative distribution function (CDF) of the SINR of the femto user equipments (FUEs) when the number of FBSs are 3, 5 and 7, respectively, and the subchannel usage ratio $\rho_{f}=1$ (i.e., the FBS utilizes entire subChannels in the operating sub-band (SB)). The proposed network model achieves higher average user SINRs for the deployment of number of FBSs equal to 3 in contrast to the deployment of number of FBSs equal to 7 and almost the same performance as depicted in Fig.5b for number of FBSs equal to 5 .

It can be seen in Fig.5 that the proposed network model efficiently increases the link performance of SUs whether in the low SINR region or in the high SINR region. The refinement of channel capacity of FUEs in Fig.3 is attributed to the two aspects: One is the superior SINR performance at cell edge for higher FRF, the other is the improvement of overall cell throughput at higher centre radius ratio.
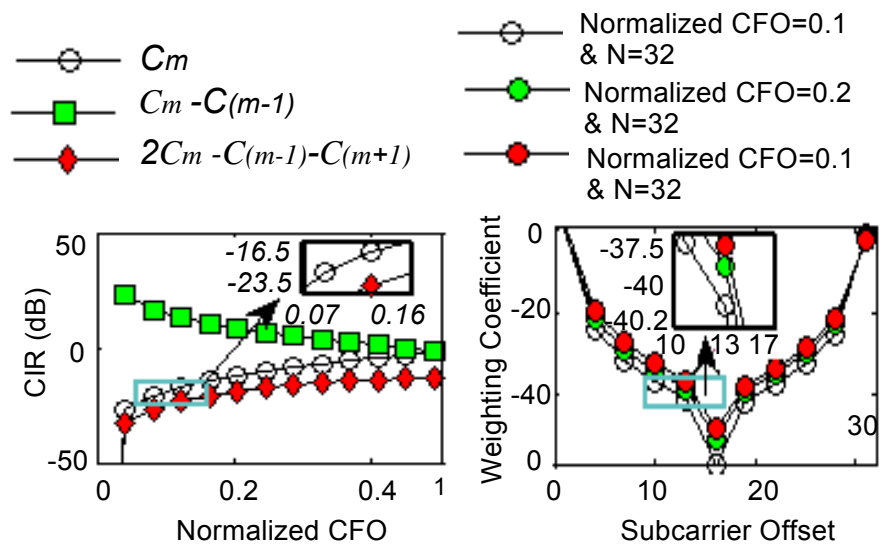

Fig. 2 Magnitude of weighting co-efficient vs. $N$ in variation of $\varepsilon$ (Fig.2a) and CIR of the weighting coefficient vs. E (Fig.2b)
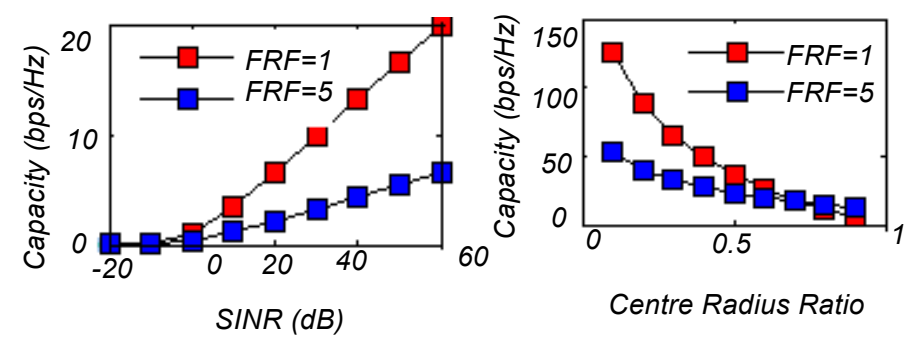

Fig.3 Variation of channel capacity with SINR for different FRF (Fig.3a) and variation of channel capacity with center radius ratio for different FRF (Fig.3b) 


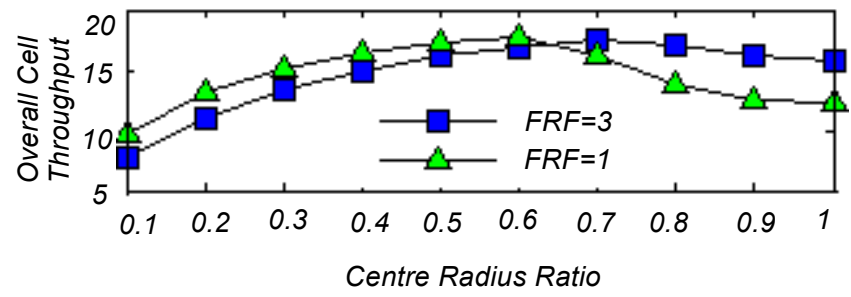

Fig.4 Cell throughput according to the center radius ratio.

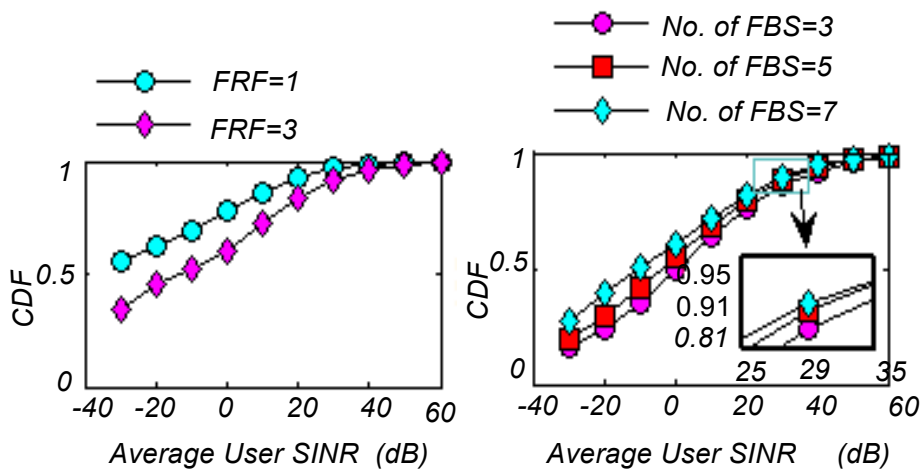

Fig.5 CDF vs. Average user SINR (dB) for different FRF (Fig.5a) and CDF vs. Average user SINR (dB) for different FRF (Fig.5b)

\section{CONCLUSIONS}

In this paper, analytical framework is developed to take on ICI caused by CFO in OFDMA system. Thereafter, a simulation testbed model is set to optimize the performance of OFDMA based cognitive radio networks by deploying more number of FBSs at macrocell edge area. The evaluation results indicate that the excellence of the optimization of the system performance at macrocell broader area are significantly affected by the large number of FBS (precisely, for more than 3 ). The optimum choice of FRF is 3 at which we can see noticeable improvement of overall cell throughput. The proposed network model is optimized up to some extent because of improvement in overall cell throughput. We efficiently address the ICI issue by compensating CFO, which also brings about the improvement of network performance.

\section{REFERENCES}

[1] V. Chandrasekhar, J. Andrews, and A. Gatherer, "Femtocell networks: A survey," IEEE Communication.Mag., vol.46, no. 9, pp.59-67, Sep.2008.

[2] FCC spectrum Policy Task Force. Report of the spectrum Efficiency Working Group, Tech. Rep. ET Docket No. 02-135, Washington DC (USA): Federal Communication Commission, 2002.

[3] Mitola J., Maguire G, "Cognitive radio: Making software radios more personal," IEEE Personal Communications, 1999, vol. 6, no. 4, p.13-18.

[4] Kollar ZS., Horvath P, "Modulation schemes for cognitive radio in white spaces," Radioengineering, 2010, vol. 19, no.4, p.511-517.

[5] Haykin S., "Cognitive radio: brain empowered wireless communications," IEEE Journal of Selected Areas in Communication, 2005, vol.23, no.2, pp.201-220.

[6] Ruttik K., Koufos K., Jantti R.,"Modeling of the secondary system's generated interference and studying of its impact on the secondary system design," Radioengineering, 2010, vol.19, no.4, pp.488-493.

[7] J Ghosh, S Dhar Roy, "Mitigating ICI at Cell Edges in CognitiveFemtocell Networks Through Fractional Frequency Reuse", IJCNDS, Vol.16, No.2, Jan.2016.
[8] C TAO, J QIU, L LIU, “A novel channel estimation algorithm with ICI mitigation over fast fading channels," Radioengineering, vol.19, no.2, June 2010,pp.347-355.

[9] M. C. Necker, "Interference Coordination in Cellular OFDMA Network," IEEE Network, Nov. Dec.2008.

[10] Defeng (David) Huang, and Khaled Ben Letaief, "Carrier Frequency Offset Estimation for OFDM Systems Using Null Subcarriers," IEEE Trans. On Communications., vol. 54, no.5, May 2006.

[11] J. Armstrong, "Analysis of new and existing methods of reducing intercarrier interference due to carrier frequency offset in OFDM," IEEE Trans. On Communications, vol. 47, pp. 365-369, Mar. 1999.

[12] Z. Wang and S. S. Abeysekera, "Frequency estimation using the pulsepair method in different fading environments," in Proc. ICASSP'03, Hong Kong, 6-10 April 2003, vol.4, pp. 517-520.

[13] Yu-Ting Sun and Jia-Chin Lin, "Synchronization for OFDM-Based Systems," National Central University, Taiwan, R.O.C, 23 August, 2011 pp-23-40.

[14] I Akbar and A.N Malik, "Performance Analysis of Carrier Frequency Offset Estimation Techniques for OFDM Systems," Research Journal of Applied Sciences, Engineering and Technology, e-ISSN: 2040-7467,2013.

[15] S. Kapoor, D. J. Marchok, and Y.-F. Huang, "Pilot assisted synchronization for wireless OFDMA systems over fast time varying fading channels," in Proc. VTC'98, 1998, pp.2077-2080.

[16] T H Stitz, T Ihalainen, A Viholainen, and M Renfors, "Pilot-Based Synchronization and Equalization in Filter Bank Multicarrier Communications," EURASIP Journal on Advances in Signal Processing, Vol.2010,Article ID 741429.

[17] Sanjay Dhar Roy, and Sumit Kundu, "Performance of an Adaptive Power Based CDMA Cognitive Radio Networks," IEEE Symposium on Industrial Electronics and Applications (ISIEA 2010), Oct 2010, Penang, Malaysia.

[18] Afaz Uddin Ahmed, Mohammad Tariqul Islam, and Mahamod Ismail, “A Review on Femtocell and its Diverse Interference Mitigation Techniques in Heterogeneous Network," Wireless Personal Communications, vol.78, no.1, March 2014.

[19] A. Damnjanovic, J. Montojo, Y. Wei, T. Ji, T. Luo, M. Vajapeyam, T. Yoo, O. Song, and D. Malladi, "A survey on 3gpp heterogeneous networks," IEEE Wireless Communication,Vol. 18,no. 3,pp.10-21,2011.

[20] Dong-Chan Oh, and Yong-Hwan Lee, "Cognitive Radio Based Resource Allocation in Femto-cell," Journal of Communications and Networks, Vol.14, No.3, June 2012.

[21] H.-W. Lee, K.-H. Han, Y.-W. Hwang and S.-H. Choi, "Opportunistic band sharing for point-to-point link connection of cognitive radios," in Proc.IEEE CrownCom, June 2009, pp. 1-5.

\section{TABLE II. SIMULATION PARAMTERS}

\begin{tabular}{|l|l|}
\hline System parameters & Values/Range \\
\hline System Bandwidth(BW) & $20 \mathrm{MHz}$ \\
\hline Macrocell Coverage Radius & $1000 \mathrm{~m}$ \\
\hline Femtocell Coverage Radius & $200 \mathrm{~m}$ \\
\hline Macrocell BS Transmit Power & $49 \mathrm{~dB}_{\mathrm{m}}$ \\
\hline Femtocell BS Transmit Power & $<20 \mathrm{~dB}_{\mathrm{m}}$ \\
\hline $\begin{array}{l}\text { Antenna Radiation Pattern of BS of Macrocell and } \\
\text { Femtocell }\end{array}$ & Omnidirectional \\
\hline Number of FBS & 12 \\
\hline Shadowing & $8 \mathrm{~dB}$ \\
\hline Angle offset & $0, \pi / 3, \pi / 6 \ldots .$. so on \\
\hline
\end{tabular}

